\title{
A REVIEW ON IMPLEMENTATION OF ALGORITHMS FOR DETECTION OF DIABETIC RETINOPATHY
}

\author{
A.P.Shingade ${ }^{1}$, A.R.Kasetwar ${ }^{2}$ \\ ${ }^{I}$ P.G student Dept.of Electronics \& Tele communication, Dr.Bhausaheb Nandurkar College of Engg \& Tech, Yavatmal \\ ${ }^{2}$ Research Scholar, Dept. of Electronics \& Tele communication, Bapurao Deshmukh College of Engg, Wardha
}

\begin{abstract}
Diabetes is a group of metabolic disease in which a person has high blood sugar. Diabetic Retinopathy (DR) is caused by the abnormalities in the retina due to insufficient insulin in the body. It can lead to sudden vision loss due to delayed detection of retinopathy. So that Diabetic patients require regular medical checkup for effective timing of sight -saving treatment. This is continuous and stimulating research area for automated analysis of Diabetic Retinopathy in Diabetic patients. A completely automated screening system for the detection of Diabetic Retinopathy can effectively reduces the burden of the specialist and saves cost as well as time. Due to noise and other disturbances that occur during image acquisition Diabetic Retinopathy may lead to false detection and this is overcome by various image processing techniques. Further the different features are extracted which serves as the guideline to identify and grade the severity of the disease. Based on the extracted features classification of the retinal image as normal or abnormal is carried out. In this paper, we have presented detail study of various screening methods for Diabetic Retinopathy. Many researchers have made number of attempts to improve accuracy, predictivity, sensitivity and specificity.
\end{abstract}

Keywords: Color Fundus Images, Diabetic Retinopathy, Exudates, Lesions.

$* * *$

\section{INTRODUCTION}

Retinopathy is due to persistent or acute damage to the retina of eye. It is an ocular manifestation of systematic disease as seen in diabetic patient called as Diabetic Retinopathy. Diabetic retinopathy is a frequent microvascular complication of diabetes and the most cause of blindness and vision loss in the working out population of the world. The risk of the disease increases with age and therefore, middle aged and older diabetic patients are prone to Diabetic Retinopathy. Usually there are no early visible symptoms of the Diabetic Retinopathy and as the disease progresses the presence of micro aneurysms, exudates both hard and soft and new blood vessels can be observed. Effective diagnosis of Diabetic Retinopathy and treatment can prevent blindness. Due to the large number of people that require screening, an automated and accurate screening tool is a useful adjunct in diabetes clinics.

Diabetic Retinopathy has four major stages:

\subsection{Mild Non Proliferative Retinopathy:}

This is the earliest stage of Diabetic Retinopathy. At this stage, micro aneurysms occur in the retina. The micro aneurysms are a tiny area of blood which is extending out from an artery or vein in the backside of the eye.

\subsection{Moderate Non proliferative Retinopathy:}

At this stage of Diabetic Retinopathy, some blood vessels that provide the nourishment to the retina are blocked. Hence there is no proper blood circulation through the blood vessels of the retina.

\subsection{Severe Non proliferative Retinopathy:}

As the disease progresses, many blood vessels from the retina are blocked due to which several area of retina is discontinued with blood supply. The demand for growing new blood vessels is then send to the body through these affected areas.

\subsection{Proliferative Retinopathy:}

This is the advance stage of Diabetic Retinopathy. At this stage, the growth of new blood vessels takes place because of the signals sent by the retina for nourishment. These newly formed blood vessels are abnormal and have thin fragile walls. If these blood vessels leak blood, severe vision loss and even blindness can result into patient.

Color Fundus Images (CFI) taken by the medical image camera by medical personnel in the hospital provides digitized data in the form of a fundus image that can be effectively used for the computerized automated detection of diabetic retinopathy

Micro aneurysms are small red dots on the retinal surface, which occur due to capillary occlusion leading to lack of 
oxygen and progression of the disease. They are less than the diameter of the optic vein .Accumulation of proteins and lipids occur in the form of exudates. Vision loss occurs when they occur in the macula.

Exudates appear as yellow or white structures in the retina. There are two types of exudates based on their appearance and occurrence. Hard exudates have well defined boundaries and the soft exudates have unclear boundaries also know as cotton wool spots [1].

Fig. 1 shows the stages of diabetic retinopathy.
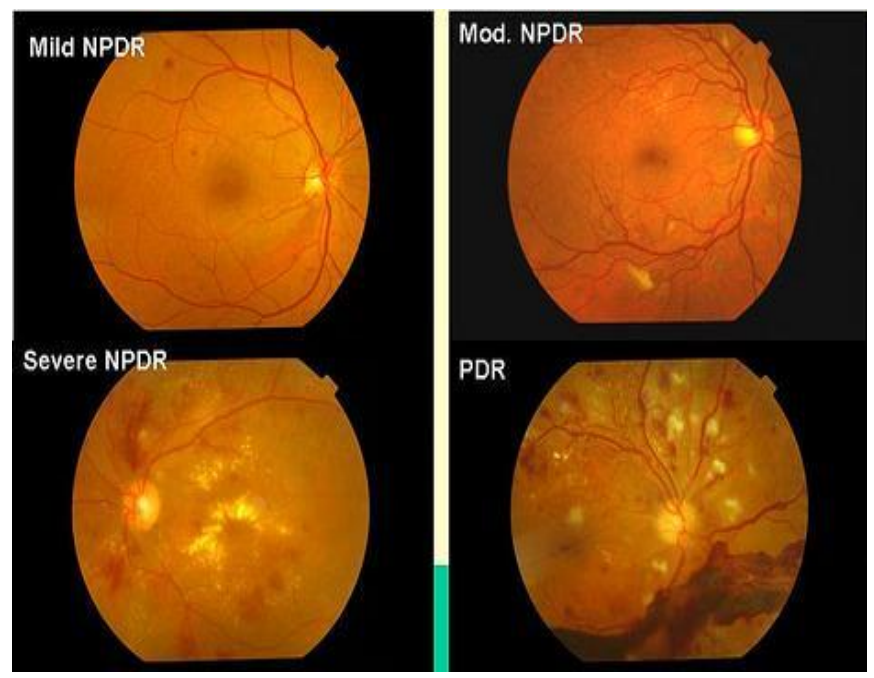

Fig-1: Stages of Diabetic Retinopathy

\section{RELATED WORK}

For detection of Diabetic Retinopathy from color fundus image various steps have been carried out. The various steps for detection of diabetic retinopathy is shown in Fig. 2.

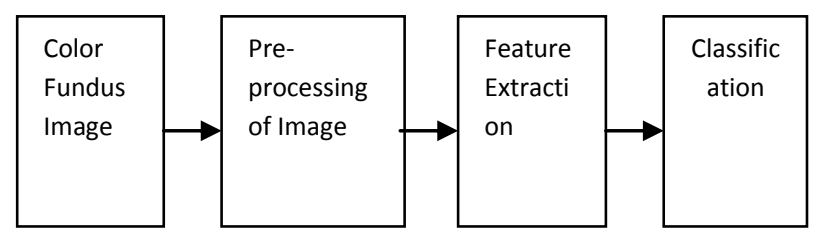

Fig. 2: Steps for detection of Diabetic Retinopathy

\subsection{Pre Processing}

For the detection of stages of Diabetic Retinopathy the Color Fundus Images are considered as an input. These images are nothing but the color images which provides the details about retina of eye. These images are preprocessed to improve the quality of image and this preprocessed image is used for the further stages.
The pixel values of Color Fundus Image are permanently distorted and the superior data is used for analysis of images. This suppresses undesired information and enhances required features. Pre processing involves brightness correction, edge detection, intensity adjustment, histogram equalization etc.

\subsubsection{Median Filtering}

The green plane image is shade corrected image is applied using a $25 \times 25$ median filter [6]. The size of the median filter was chosen such that it is wider than the widest blood vessel in used dataset of images. The shade corrected image has negative values for all pixels which have intensity lower than the background. By removing all pixels with a positive value from the image, bright pathologies no longer influence the later analysis. All pixels with a positive value were set to zero resulting in the preprocessed image. The green plane of the image (I) is filtered using a 3x 3 median filter to remove occasional salt-and-pepper noise [7]. Further noise removal is performed by convolving the image with gauss (2). The background intensity $(\beta)$ of filtered image is estimated by applying 65 x 65 median filtering. So the shade corrected image is obtain by $S^{\prime}=(I / \beta)-1$

The green plane image is shade corrected image is applied using a 56x56 median filter [9]. A top hat transform is applied using a 25 pixel long linear structuring element which is spaced 180 degrees apart. Two matched filters are used to approximate the characteristics of the objects of different sizes and each is threshold and combined. The result is a micro aneurysm seed image. The region growing process is carried out using the seed pixel from which eighty features are extracted. A series of image processing techniques were used in the method proposed by Atushi Mizutani et al [18] which includes brightness correction, gamma correction and contrast enhancement. Median filtering is employed to reduce the effect of noise.

\subsubsection{Morphological Transformation}

Pre-processing is carried out for detection of Dark lesions which have very low content in the green colour plane [10]. Moreover the background intensity variation of the image in the green plane is less. Hence the green channel of an image is obtained initially and for every pixel $p$ in the image, a neighborhood is considered centered on the selected pixel. If the gray level of the pixel is lesser than the fraction of the mean of then the pixel is considered as darker than the surrounding pixels. The darker areas than the normal retina are detected. Finally a binary image is obtained with non zero values for selected dark pixels. In the method proposed by Meindert Niemeijer et.al [11], the green plane of the image undergoes shade correction. The shade corrected image is top hat transformed to segment the vessels present in the image. In order to eliminate the blood vessels a vascular tree algorithm is proposed [13].The vascular tree is considered as the only part of the image that is uniform throughout. 
Morphological opening with a linear structuring element of size 15 pixels is used to remove rounded bright zones of size less than 15 pixels. The contrast is improved by obtaining the sum of the top hat transforms. The resulting images are noisy and Gaussian smoothing is used to eliminate the noise present in the image. To completely eliminate dark lesions, a linear opening by reconstruction of size 15, a linear closing by reconstruction of size 15 , and linear opening of size 29 is done alternatively. The reconstructed image is subtracted from the shade corrected image; matched filter of size $11 \times 11$ pixels is then used. A threshold value is set which results in a binary image. The binary image is not a replica of the original image as a result region growing is done using the darkest pixel as the starting point. Akara Sopharak et al in their work to detect exudates made use of morphological filtering. The preprocessing of image involves the conversion in RGB space to Hyper spectral imaging (HSI) colour space, as the intensity components are removed from the remaining colour components. Contrast adaptive histogram equalization is applied to small regions of the image. The small regions are combined by bilinear interpolation after equalization. Exudates are regions of high intensity values [14].

\subsubsection{Histogram Equalization}

A neural network based preprocessing stage for histogram equalization and specialization is utilized [15]. A sliding window technique uses the regions covered by the window serve as the input features. For preprocessing, the green plane shaded colour images are considered. If the exudates are the center pixel then it is considered as positive sample else it is considered as negative sample. To locate the exudates, a 9x9 sized window is used. A target vector is created based on the positive and negative samples and the vector is set as 0 or 1 .

\subsubsection{Radon Cliff Operator}

The detection of micro aneurysms with the help of Radon Cliff operator is presented by Luca Giancardo et al [20]. In this work, the pre-processing involves the analysis of discrete stationary wavelets which performed in the green plane of the image. A signed image is obtained by reconstructing the image by replacing first scale plane with zeros. This removes the background of image. The negative pixels are then extracted and normalised. The optic nerve, bright lesions are eliminated enhancing the micro aneurysms and vessels. The radon cliff operator is applied to the gray scale image and for pixel values greater than 215 are considered as candidates.

\subsubsection{Thresholding}

S.Kavitha et al proposed a method for detection of hard and soft exudates wherein the preprocessing involves colour space conversion into lab colour space and detection of fundus region [25]. Further the Lab colour space image is converted into binary image by thresholding. The binary image is morphologically closed and a fundus mask is created with pixels at the fundus marked as 1 and pixels in the background marked as 0 .

\subsection{Micro Aneurysms Detection}

For the effective detection of Diabetic Retinopathy in Diabetic patients Microaneurysms (MA) are detected as these are the earliest sign of the Diabetic Retinopathy.

\subsubsection{Double Ring Filter}

After image pre-processing, candidate regions for microaneurysms were detected using a double-ring filter [18]. The diameters of 5 and 13 pixels for inner and outer ring respectively is used for detecting micro aneurysms in the surrounding retinal regions of the image. Any potential false positives located in the regions corresponding to blood vessels were removed by automatic extraction of blood vessels from the images. One hundred twenty six image features were determined and 28 components were selected by using principal component analysis. The candidate lesions were classified into microaneurysms or false positives using the rule-based method and an artificial neural network.

\subsubsection{Morphological Filtering}

Morphological closing leads to twisted vessel like patterns being treated as MA, an ideal operator with all structuring elements having large diameter is used [26]. The binary features, gray level and colour features are extracted for the detection of MAs.

\subsubsection{Density Function}

The spatial relationship of the pixels has considered to differentiate between the candidate lesions, noise and pigment variability. A density function is defined to find the number of similar pixels in the neighborhood of the selected pixel. A threshold value is set to eliminate pixels belonging to the optic disk and the blood vessels as a result the dark lesions are identified [24].

\subsubsection{Region Growing}

Region growing algorithm is used to find the morphology of the candidate. The features are defined to further improve the accuracy of detection in the method [27]. A modification is made so that the measures were multiplied with a scale factor for better discrimination between micro aneurysms and other parts of the vasculature.

\subsubsection{Local Rotating Cross-Section Profile}

Microaneurysm detection through the analysis of directional cross-section profiles is proposed by Istvan Lazar et al. [33], centered on the local maximum pixels of the preprocessed image. The peak detection is applied on each profile and then a set of attributes regarding the size, height, and shape of the 
peak are calculated. The statistical measures of these attribute values, as the orientation of the cross-section changes; constitute the feature set that is used to exclude spurious candidates.

\subsection{Exudates Detection}

Exudates are any fluid that filters from circulatory system of eye into lesions or areas of inflammation.

\subsubsection{Fuzzy C-Means Clustering}

Fuzzy C-means clustering is used to detect exudates based on four features and eight clusters are formed for 40 retinal images. The original image with the exudates region absent is then subtracted from the original image to find the areas where exudates are present. The cluster image is used as marker and original image as mask and morphological reconstruction by dilation is carried out. Then thresholding is applied and difference between the original and reconstructed image is found. The resulting image is superimposed on the original retinal image. Akara Sopharak et al used fuzzy C-means clustering for the detection of exudates in retina images. In the pre-processing stage the image the exudates are distinguished by means of the intensity level .The image is converted from the RGB colour space to Hyper Spectral Image (HSI) colour space and median filtering is applied to the I plane of the image and a contrast enhanced adaptive histogram equalisation is applied [19]. The standard deviation of the contrast enhanced image is used as one of the features for detecting the exudates. The hue of the HSI image is the next feature to be extracted. The number of edge pixels is the final feature for clustering. The optic disc is removed by using an entropy feature. To ensure the neighboring pixels are included, a dilation process is carried out using a disc structuring element .Then the number of white pixels in the image are found [16].

\subsubsection{K Means Clustering}

$\mathrm{K}$ means clustering is used to detect exudates and non exudates as a result the number of clusters formed. Exudates are located in high intensity range whereas the other lesions are present in the low intensity range. Maximum and minimum intensity values are calculated and serve as a distance measure. The process is repeated and the cluster centers are updated in each step [22].

\subsubsection{Neural Network System}

Neural networks are used for the detection of exudates [27]. The pre processing is done in the entire colour plane unlike other methods that consider only the green plane and then applying histogram equalisation to remove the non uniform lighting conditions. A sliding window mask is moved over the entire pixel region such that each pixel falls in the center of the mask.

\subsubsection{Contextual Clustering}

The contextual information is used to enhance the detection rate of exudates. Fuzzy art neural network [28] is also used for the detection of exudates. From the contrast enhanced image features such as convex area, solidity and orientation are extracted. The optic disc is eliminated based on the fact that it is the object with the largest diameter in the image. Classification is based on four features namely convex area, convex image.

\subsubsection{Linear Discriminant Analysis}

The detection method of hard exudates based on Fisher's linear discriminant analysis is developed by Clara I.Sanchez et al. Meindert Niemeijer et al [12] in their work on the automatic detection of diabetic retinopathy proposed a method for the early detection of exudates, cotton wool spots and drusens.

\subsection{Classifier}

The classification task consists of deriving a general classification rule for unknown candidates from the training set. The method for the classification should be robust against outliers in the training set, because it is very difficult to obtain an absolutely reliable ground truth and the distribution of the features. There exist many classifiers for the detection of MA such as support vector machines, k-nearest - neighbor (KNN) method, linear discriminant analysis, Gaussian filter and neural network have been used.

For the analysis, A Linear discriminant method was used as an effective classification and all methods that provided better results were taken into consideration. It is found that AD Treea tree based classifier, SVM classifier and Logistic Regression were used and the performance is analyzed. 15 features are extracted from 115,867 positive and negative samples of exudates pixels. Classification is made using the Naïve Bayes classifier which repeatedly removes the features till the performance of classifier does not improve. The previously removed features are also considered and some features are found by Bayes classifier. By using these features, SVM classifier is trained. For each pair of parameters, tolerance and radial basis function is determined [27].

For the classification of exudates, 15 features are extracted from the image namely the pixel intensity, standard deviation, hue, number of edge pixels, average intensity of pixel's cluster, size of cluster, average intensity of pixels in the neighborhood of pixels, distance between pixel cluster and optic disc, filter response of 6 difference of Gaussian filters. Bayes classifier is used for the classification [12].

The KNN classifiers, which consist of assigning the class to a new candidate $(\mathrm{X})$ to which the majority among the $\mathrm{K}$ nearest neighbours of $(\mathrm{X})$ belongs. The advantages of KNN classifiers 
are the method becomes robust against outliers and it is nonparametric (i.e. no assumption about the feature distribution must be made) for reasonably large value of $\mathrm{K}$. One drawback of KNN classifiers is that all neighbours have the same weight for the decision, independently of their distance from the candidate to classify. Moreover, if the training set is very asymmetric for the two classes particularly for large $\mathrm{K}$ the method might be failed. In order to overcome these problems and to meet the above requirements, the kernel method is chosen for density estimation, combined with Bayesian risk minimization [6].

Three layered perceptron architecture, i.e. a single input layer, single hidden layer and single output layer neural network is used [15]. The input consists of 243 neurons, 50 hidden neurons and output consists of a single neuron. A scaled conjugate gradient method was used. If the neural network classifier is used then the output of a network is in the range of 0 to 1 . A neural network classifier with multilayer perceptron is used [23]. A log-Sigmoid transfer function in the output layer and linear function in the output layer is employed which makes use of back propagation algorithm.

\section{SUMMARY \& DISCUSSION}

As discussed in previous section it has been noted that the various classifiers were employed by existing methods and performance was evaluated. Different values of the parameters such as accuracy, sensitivity, positive predictive value (PPV) and specificity were obtained which exclusively determined effective detection of Diabetic Retinopathy.

\subsection{Limitations of the Current Approaches}

An approach for the detection of diabetic retinopathy from retinal image involves the identification of Micro aneurysms and exudates from retinal features such as the optic disc, area of blood vessels and fovea and localizing the necessary structures whereas Colored retinal structures are quite difficult to locate. The classification was carried out for presence or absence of Diabetic Retinopathy only. The existing systems are more complex and are time consuming as the normal computers executing time to perform operation is quite high.

\subsection{Future Scope for Research}

The effective screening of retinal images of Diabetic patients for the detection of Diabetic Retinopathy and classification of different stages of Diabetic Retinopathy can reduce the possibilities of sudden vision loss in affected patients. As the existing systems are quite slow in operation, a real time implementation of screening system can provide the better performance. For such implementation on real time platform DSPs or FPGAs can be preferred as it can provide the advanced results for the detection of Diabetic Retinopathy. Such screening systems for the detection of different stages of Diabetic Retinopathy mainly benefit affected patients from rural areas who are mostly unaware of the presence of the Diabetic Retinopathy.

Table -1: Comparative analysis of Performance Measures

\begin{tabular}{|c|c|c|c|c|c|}
\hline $\begin{array}{l}\text { Ref. } \\
\text { No. }\end{array}$ & $\begin{array}{l}\text { Feature } \\
\text { Extract } \\
\text { ed }\end{array}$ & $\begin{array}{l}\text { Techniques } \\
\text { used for } \\
\text { preprocessing }\end{array}$ & $\begin{array}{l}\text { Techniques } \\
\text { used for feature } \\
\text { extraction }\end{array}$ & $\begin{array}{l}\text { Classifier } \\
\text { Used }\end{array}$ & Performance Measured \\
\hline 2 & Lesions & $\begin{array}{l}\text { Median } \\
\text { filtering }\end{array}$ & $\begin{array}{l}\text { Morphological } \\
\text { Filtering }\end{array}$ & $\begin{array}{l}\text { K-Nearest } \\
\text { Neighbour }\end{array}$ & $\begin{array}{l}\text { Sensitivity-100\% } \\
\text { Accuracy- } 87 \%\end{array}$ \\
\hline 3 & $\begin{array}{l}\text { Micro } \\
\text { aneurys } \\
\text { ms }\end{array}$ & $\begin{array}{l}\text { Median } \\
\text { filtering }\end{array}$ & $\begin{array}{l}\text { Double ring } \\
\text { filter }\end{array}$ & Not Used & $\begin{array}{l}\text { Sensitivity-85.4\% } \\
\text { Accuracy- } 83.1 \%\end{array}$ \\
\hline 8 & $\begin{array}{l}\text { Exudat } \\
\text { es }\end{array}$ & $\begin{array}{l}\text { Histogram } \\
\text { Equalization }\end{array}$ & $\begin{array}{l}\text { Fuzzy C-means } \\
\text { Clustering }\end{array}$ & Not Used & $\begin{array}{l}\text { Sensitivity-80\% } \\
\text { Specificity-99.5\% }\end{array}$ \\
\hline 9 & $\begin{array}{l}\text { Exudat } \\
\text { es }\end{array}$ & $\begin{array}{l}\text { Morphological } \\
\text { Transformatio } \\
\mathrm{n}\end{array}$ & $\begin{array}{l}\text { Neural } \\
\text { Network } \\
\text { System }\end{array}$ & $\begin{array}{l}\text { Neural } \\
\text { Network }\end{array}$ & $\begin{array}{l}\text { Sensitivity-94.78\% } \\
\text { Specificity- } 94.29 \%\end{array}$ \\
\hline 12 & $\begin{array}{l}\text { Micro } \\
\text { aneurys } \\
\text { ms }\end{array}$ & Thresholding & $\begin{array}{l}\text { Double ring } \\
\text { filter }\end{array}$ & Not Used & Sensitivity-68\% \\
\hline 13 & $\begin{array}{l}\text { Micro } \\
\text { aneurys }\end{array}$ & $\begin{array}{l}\text { Radon Cliff } \\
\text { operator }\end{array}$ & $\begin{array}{ll}\text { Radon } & \text { Cliff } \\
\text { operator } & \end{array}$ & $\begin{array}{l}\text { Support } \\
\text { Vector }\end{array}$ & Sensitivity-41\% \\
\hline
\end{tabular}




\begin{tabular}{|c|c|c|c|c|c|}
\hline & $\mathrm{ms}$ & & & Machine & \\
\hline 15 & $\begin{array}{l}\text { Exudat } \\
\text { es }\end{array}$ & $\begin{array}{l}\text { Morphological } \\
\text { Transformatio } \\
\mathrm{n}\end{array}$ & $\begin{array}{l}\mathrm{K} \text { means } \\
\text { clustering }\end{array}$ & Not Used & $\begin{array}{l}\text { For GFC photographs - } \\
\text { Sensitivity-90.24\% and } \\
\text { PPV- } 89.75 \% \\
\text { For Angiographs } \\
\text { Sensitivity-93.74\% and } \\
\text { PPV- } 91.67 \% \\
\text { For Color photographs- } \\
\text { Sensitivity-89.62\% and } \\
\text { PPV- } 89.50 \%\end{array}$ \\
\hline 20 & $\begin{array}{l}\text { Exudat } \\
\text { es }\end{array}$ & $\begin{array}{l}\text { Region } \\
\text { Growing }\end{array}$ & $\begin{array}{l}\text { Neural } \\
\text { Network } \\
\text { System }\end{array}$ & $\begin{array}{l}\text { Support } \\
\text { Vector } \\
\text { Machine }\end{array}$ & Accuracy $-98.77 \%$ \\
\hline 24 & $\begin{array}{l}\text { Micro } \\
\text { aneurys } \\
\text { ms }\end{array}$ & Thresholding & $\begin{array}{l}\text { Local Rotating } \\
\text { Cross-Section } \\
\text { Profile }\end{array}$ & $\begin{array}{l}\text { Gaussian } \\
\text { Filter }\end{array}$ & $\begin{array}{l}\text { Sensitivity at false positive } \\
\text { rates } 1 / 8 \text { and } 1 / 4 \text { FPs image }\end{array}$ \\
\hline 26 & $\begin{array}{l}\text { Exudat } \\
\text { es }\end{array}$ & $\begin{array}{l}\text { Morphological } \\
\text { Reconstructio } \\
\mathrm{n}\end{array}$ & $\begin{array}{l}\text { Fuzzy C-means } \\
\text { Clustering }\end{array}$ & Not Used & $\begin{array}{l}\text { Sensitivity-87.28\%, } \\
\text { Accuracy-99.11 } \\
\text { Specificity-99.24\% } \\
\text { PPV-42.77\% } \\
\text { PLR- } 224.26 \\
\end{array}$ \\
\hline
\end{tabular}

\section{REFERENCES}

[1]. Diabetic Retinopathy", U.S. DEPARTMENT OF HEALTH AND HUMAN SERVICES National Institutes of Health National Eye Institute NIH Publication No: 062171Revised 9/03.

[2]. Thomas Walter, Jean-Claude Klein, Pascale Massin, and Ali Erginay, "A Contribution of Image Processing to the Diagnosis of Diabetic Retinopathy-Detection of Exudates in Color Fundus Images of the Human Retina”, IEEE Transactions On Medical Imaging, Vol. 21, No. 10, October 2002.

[3]. "A Novel Approach to Diagnose Diabetes Based on the Fractal Characteristics of Retinal Images", Shu-Chen Cheng and Yueh-Min Huang,IEEE Transactions On Information Technology In Biomedicine, Vol. 7, No. 3, September 2003. [4]. "Automated Feature Extraction in Color Retinal Images by a Model Based Approach", Huiqi Li and Opas Chutatape, IEEE Transactions On Biomedical Engineering, Vol. 51, No. 2, February 2004.

[5]. "Ridge-Based Vessel Segmentation in Color Images of the Retina", Joes Staal, Michael D. Abràmoff, Meindert Niemeijer, Max A. Viergever, and Bram van Ginneken, IEEE Transactions On Medical Imaging, Vol. 23, No. 4, April 2004. [6]. Meindert Niemeijer, Bram van Ginneken, Joes Staal, Maria S. A.Suttorp-Schulten, and Michael D. Abràmoff, "Automatic Detection of Red Lesions in Digital Color Fundus Photographs", IEEE transactions on medical Engineering, vol. 24, no. 5, May 2005 .
[7]. "Automated Microaneurysm Detection Using Local Contrast Normalization and Local Vessel Detection", Alan D. Fleming, Sam Philip, Keith A. Goatman, John A. Olson, and Peter F. Sharp IEEE Transactions on medical imaging, vol. 25, no. 9, September 2006.

[8]. Thitiporn Chanwimaluang, Guoliang Fan, and Stephen R. Fransen, "Hybrid Retinal Image Registration", IEEE Transactions On Information Technology In Biomedicine, Vol. 10, No. 1, January 2006.

[9]. "Integrated Analysis of Vascular and Nonvascular Changes from Color Retinal Fundus Image Sequences Harihar Narasimha-Iyer, Ali Can, Badrinath Roysam, Howard L. Tanenbaum, and Anna Majerovics, IEEE Transactions on biomedical engineering, vol. 54, no. 8, August 2007.

[10]. "Detection of Anatomic Structures in Human Retinal Imagery",Kenneth W. Tobin, Edward Chaum, V. Priya Govindasamy, and Thomas P. Karnowski, IEEE Transactions on medical imaging, vol. 26, no. 12, December 2007.

[11]. "Segmentation of the Optic Disc, Macula and Vascular Arch in Fundus Photographs", Meindert Niemeijer, Michael D. Abràmoff and Bram van Ginneken, IEEE Transactions on medical imaging, vol. 26, no. 1, January 2007.

[12]. .Meindert Niemeijer, Bram van Ginneken,Stephen R. Russell,Maria S. A. Suttorp-Schulten,5and Michael D. Abra'moff , "Automated Detection and Differentiation of Drusen,Exudates, and Cotton-Wool Spots in Digital Color Fundus Photographs for Diabetic Retinopathy Diagnosis",IOVS, Vol.48, No. 5, May 2007. 
[13]. "Optimal Wavelet Transform for the Detection of Microaneurysms in Retina Photographs", Gwénolé Quellec, Mathieu Lamard, Pierre

Marie Josselin, Guy Cazuguel,Béatrice Cochener, and Christian Roux, IEEE Transactions on medical imaging, vol. 27, no. 9, September 2008.

[14]. Akara Sopharak, Bunyarit Uyyanonvara , Sarah Barmanb, Thomas H. Williamson, "Automatic detection of diabetic retinopathy exudates from non dilated retinal images using mathematical morphology methods",Computerised Medical Imaging and Graphics,Vol.32,pp 720-727,2008.

[15]. Gerald Schaefer and Edmond Leung, "An investigation into neural network for detection of exudates in retinal images", Applications of Soft Computing, ASC 52, pp. 169 177.2009.

[16]. "A Computational-Intelligence-Based Approach for Detection of Exudates in Diabetic Retinopathy Images, "Alireza Osareh, Bita Shadgar, and Richard Markham, IEEE Transactions on information technology in biomedicine, vol. 13, no. 4, july 2009.

[17]. "Information Fusion for Diabetic Retinopathy CAD in Digital Color Fundus Photographs", Meindert Niemeijer, Michael D. Abràmoff, and Bram van Ginneken, IEEE Transactions on medical imaging, vol. 28, no. 5, may 2009.

[18]. Atsushi Mizutani,Chisako Muramatsu,Yuji Hatanaka," Automated Microaneurysm detection method based on double-ring filter in retinal fundus images" IEEE Transactions on Medical imaging, Proc. of SPIE Vol. 7260, 72601N 1$5,2009$.

[19]. "Automatic exudates detection form non-dilated Diabetic Retinopathy using Fuzzy C-means clustering”, Sensors, Vol.9, pp.2148- 2161, 2009.

[20]. Luca Giancardoa,b, Fabrice Meriaudeaub, Thomas P. Karnowskia, Kenneth W. Tobina, Yaqin Lic and Edward Chaum, M.D., "Microaneurysms detection with Radon Cliff operator in retinal fundus images", IEEE Transactions on Medical Imaging ,Proc. Of SPIE Vol. 7623, 2010.

[21]. "Multiscale AM-FM Methods for Diabetic Retinopathy Lesion Detection", Carla Agurto, Victor Murray, Eduardo Barriga, Sergio Murillo, Marios Pattichis, Herbert Davis, Stephen Russell,Michael Abràmoff, and Peter Soliz. IEEE Transactions on medical imaging, vol. 29, no. 2, February 2010.

[22]. "A Successive Clutter-Rejection-Based Approach for Early Detection of Diabetic Retinopathy”,Keerthi Ram, Gopal Datt Joshi, and Jayanthi Sivaswamy, IEEE Transactions on biomedical engineering, vol. 58, no. 3, march 2011.

[23]. Gardner GG, Keating D, Williamson TH, Elliott AT, "Automatic detection of diabetic retinopathy using an artificial neural network: a screening tool", British journal of Ophthalmology, pp. 940-944, 2011.

[24]. Xiayu Xu, Meindert Niemeijer, Qi Song, Milan Sonka et.al., "Vessel Boundary Delineation on Fundus Images Using Graph-Based Approach", IEEE Transactions on medical imaging, vol. 30, no. 6, June,2011.
[25]. S.Kavitha and K.Duraiswamy,"Automatic detection of hard and soft exudates in fundus images using colour histogram thresholding",European Journal of Scientific Reasearch,Vol.48,No.3,pp.493-504,2011.

[26]. Diego Marín, Arturo Aquino, Manuel Emilio GegúndezArias, and José Manuel Bravo "A New Supervised Method for Blood Vessel Segmentation in Retinal Images by Using GrayLevel and Moment Invariants-Based Features", IEEE Transactions on medical imaging, vol. 30,no. 1, January 2011. [27]. "Optic Disc Detection From Normalized Digital Fundus Images by Means of a Vessels' Direction Matched Filter", Aliaa Abdel-Haleim Abdel-Razik Youssif, Atef Zaki Ghalwash, and Amr Ahmed Sabry Abdel-Rahman Ghoneim, IEEE Transactions on medical imaging, vol. 27, no.1, January 2008 .

[28]. "Automated Measurement of the Arteriolar-to-Venular Width Ratio in Digital Color Fundus Photographs",Meindert Niemeijer, Xiayu Xu,Alina V. Dumitrescu, Priya Gupta, Bram van Ginneken, James C. Folk, and Michael D. Abrámoff, IEEE Transactions on medical imaging, vol.30, no. 11, november 2011.

[29]. K. Sai Deepak and Jayanthi Sivaswamy, "Automatic Assessment of Macular Edema from Color Retinal Images", IEEE Transactions on medical imaging, vol. 31, no. 3, March 2012.

[30]. Balazs Harangi, Balint Antal, Andras Hajdu, "Automatic Exudates Detection with Improved Naïve Bayes Classifier", IEEE transactions on Medical Imaging, pp.61-65, 2012.

[31]. "An Ensemble-Based System for Microaneurysm Detection and Diabetic Retinopathy Grading”, B'alint Antal, and Andr'as Hajdu, IEEE Transactions On Biomedical Engineering, Vol. 59, No. 6, June 2012.

[32]. "A Review Of Automated Diabetic Retinopathy Diagnosis From Fundus Image", K.NARASIMHAN, 2 NEHA.V.C, 3 K.VJAYAREKHA. Journal of Theoretical and Applied Information Technology 15 May 2012. Vol. 39 No.2 [33. Istvan Lazar and Andras Hajdu, "Retinal Microaneurysm Detection Through Local Rotating Cross-Section Profile Analysis", IEEE transactions on medical imaging, vol. 32, no. 2, February 2013.

[34]. "Assessing the Need for Referral in Automatic Diabetic Retinopathy Detection", Ramon Pires, Herbert F. Jelinek, Jacques Wainer, Siome Goldenstein, Eduardo Valle, and Anderson Rocha, IEEE Transactions on medical imaging, vol. 32, no. 2, February 2013.

[35]. Nutnaree Kleawsirikul, Smith Gulati, and Bunyarit Uyyanonvara, "Automated Retinal Hemorrhage Detection Using Morphological Top Hat and Rule-based Classification". 3rd International Conference on Intelligent Computational Systems (ICICS'2013) April 29-30, 2013 Singapore. 


\section{BIOGRAPHIES}
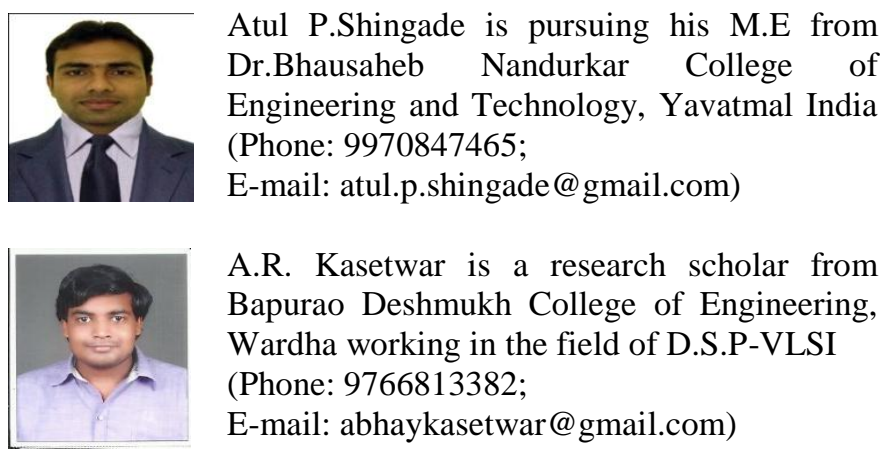

A.R. Kasetwar is a research scholar from Bapurao Deshmukh College of Engineering, Wardha working in the field of D.S.P-VLSI (Phone: 9766813382;

E-mail: abhaykasetwar@gmail.com) 